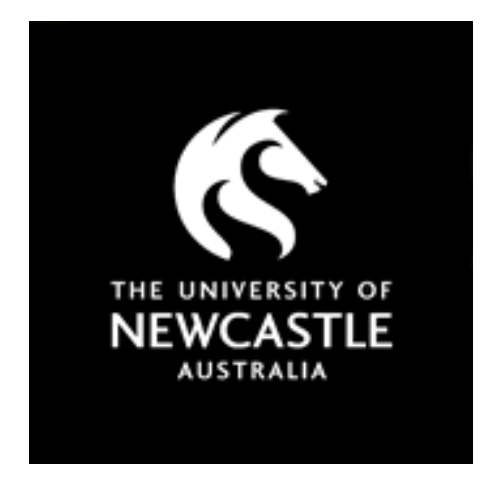

NOVA

University of Newcastle Research Online

nova.newcastle.edu.au

Hollis, Jenna L.; Williams, Lauren T.; Collins, Clare E.; Morgan, Philip J. "Does motivational interviewing align with international scope of practice, professional competency standards, and best practice guidelines in dietetics practice?", Journal of Academy of Nutrition and Dietetics Vol. 114, Issue 5, p. 676-687 (2012)

Available from: http://dx.doi.org/10.1016/j.jand.2013.12.023

Accessed from: http://hdl.handle.net/1959.13/1044149 


\section{Does Motivational Interviewing align with international scope of practice, professional competency standards and best practice guidelines in dietetic practice?}

\section{Abstract}

1 The dietetic profession has become increasingly interested in identifying effective counseling

2 models, particularly for situations when advice-giving is unsuccessful. Motivational

3 interviewing (MI) is a directive, client-centered counseling style that aims to facilitate

4 behavior change by creating a neutral, non-judgmental environment where the client can

5 explore and resolve ambivalence to behavior change. A challenge of incorporating any

6 counseling approaches into dietetic practice is ensuring that scope of practice and

7 professional standards are maintained whilst also employing the most effective and client-

8 preferred counseling method. This paper aims to (i) examine how MI fits within international

9 dietetic scope of practice, competency standards, and evidenced-based guidelines and (ii)

10 provide recommendations for applying MI in dietetic practice and research. United States,

11 Canadian, European and Australian dietetic scope of practice statements, professional competency standards and evidenced-based weight management guidelines were examined for congruency with MI principles and clinical strategies. Two of the four core MI concepts, partnership and compassion, were consistent with dietetic scope of practice statements and competency standards. Reference to acceptance was found in European and Canadian standards, while evoking intrinsic motivation was absent from all professional standards. The majority of MI strategies were supported by international evidenced-based guidelines, however implementation may not be MI-adherent if conducted before the client has expressed a genuine commitment to change. Training in MI is compatible with international dietetic practice competency standards and evidenced-based practice recommendations and

21 could be a valuable asset to dietetic practice. 


\section{Does Motivational Interviewing align with international scope of practice, professional competency standards and best practice guidelines in dietetic practice?}

\section{Introduction}

Facilitating change in client behavior is a key challenge for dietitians. Practitioner frustration occurs when clients do not adhere to the behavior change plans developed during consultations. ${ }^{1}$ The client may return for future appointments having made minimal or no lifestyle changes, or showing a decline in diet and physical activity behaviors. Despite many clients having the knowledge, skills and need to make nutritious dietary choices and to participate in regular physical activity, many still struggle to maintain behavior change in the long-term. ${ }^{2}$ Clients may feel ambivalent at the need to make behavior changes. ${ }^{3}$ Ambivalence is a state of mixed feelings resulting in an inability to choose between two courses of action. ${ }^{3}$ When confronted with feelings of ambivalence in clients, the dietitian may take the role of arguing for change, hoping to convince their client of the benefits of changing behavior. In response, the client may feel judged and criticized, and may rationalize their current behavior by providing arguments to maintain the status quo, stop engaging with the dietitian or may silently resolve not to change. ${ }^{4}$

Dietitians have traditionally been trained to facilitate behavior change through advice-giving, taking primary responsibility for setting the agenda and direction of consultations. ${ }^{5}$ An advice-giving consultation predominantly draws on an 'informing' communication style, where the dietitian is regarded as the expert in the client's health and persuades the client to change, whilst the client listens to the advice. ${ }^{3}$ There is some evidence that this communication style may be effective where the client prefers this style of consultation or if 
43 the client is unaware of the appropriate treatment. ${ }^{6,7}$ However, an 'informing' style may be

61 Motivational Interviewing (MI) ${ }^{13,14}$ is a directive, client-centered counseling style that aims to met with resistance if it involves conveying information that the client is already aware of, has previously tried to act on or disagrees with, and fails to enquire about the client's opinions and concerns, negotiate treatment options or consider readiness to change., ${ }^{4,6}$

The dietetic profession has become increasingly interested in identifying effective counseling theories $^{8}$, particularly for situations when advice-giving is ineffective. ${ }^{9,10}$ There is evidence that a dietitians communication skills can influence client outcome ${ }^{11}$ and satisfaction. ${ }^{10}$ Hancock and colleagues ${ }^{7}$ found that the majority of clients reported feeling valued by the dietitian when they believed that they had been listened to on the basis of the dietitian paraphrasing and using reflective statements. Rapport, empathy, delivery of effective and reliable information and providing a non-judgmental environment were valued by the client, who reported that these factors created an environment where they would be more likely to talk openly. ${ }^{7}$ In a 2011 survey of Australian dietitians ${ }^{12}$, 47\% reported that they lacked adequate counseling skills for behavior change to provide effective obesity treatment, with $59 \%$ of respondents indicating interest in developing these skills through continuing professional development programs.

62 facilitate behavior change by creating a neutral, non-judgmental environment where the client feels comfortable to explore ambivalence to behavior change. ${ }^{3,15}$ MI supports a guiding communication style and aims to strengthen a client's commitment to behavior change by: 1) developing a collaborative partnership between the health professional and client, 2) evoking 
66 intrinsic motivation, 3) providing compassion and 4) showing acceptance (table 1). ${ }^{15}$ MI

67 advocates a range of communication skills and clinical strategies that facilitate the discussion of ambivalence, enable the health professional to elicit and selectively reinforce client change talk to enhance motivation and respond to any resistance encountered in a way that intends to reduce it. $^{3,15}$

Studies have shown MI to be effective for weight loss and reducing systolic blood pressure, total blood cholesterol and blood alcohol concentration. ${ }^{13,14}$ Whilst research into the effectiveness of MI for diet and physical activity behavior change has been conducted using psychologists $^{16}$, counselors ${ }^{17}$ and nurses ${ }^{18}$, few have employed dietitians. As a result, the efficacy and cost-effectiveness of MI in dietetic practice has not yet been established. ${ }^{19}$ In one study in dietitians, Bowen and colleagues $^{20}$ reported a significant reduction in the percentage energy from fat consumed by women receiving a one year counseling intervention from an MI-trained dietitian compared to a standard care group $(-1.3 \%$ vs. $+1.4 \%$, $\rho<0.001) .{ }^{20}$ However, this study did not use validated techniques to assess the competency of 81 dietitians providing the MI counseling. ${ }^{20}$ Brug and colleagues ${ }^{11}$ found a significantly lower saturated fat score in a group that received MI counseling by a dietitian, compared with a group counseled by a dietitian not trained in $\mathrm{MI}(\beta=0.23, \rho<0.01)$. They did assess MI fidelity, and found that despite the MI-trained dietitians not attaining MI competency, they were more empathetic, reflected more frequently and were more likely to allow their clients to speak for the majority of the consultation. ${ }^{11}$ 
88 A challenge of incorporating MI into dietetic practice is ensuring that scope of practice and professional standards are maintained within an effective and client-preferred counseling approach. ${ }^{9}$ Achieving proficiency relies to some extent on whether the values and beliefs of the professional group align with the MI counseling framework. ${ }^{21}$ To the authors’ knowledge, no papers have examined how MI aligns with the scope of practice or competency standards of the dietetic profession. Therefore, the aims of this paper are to (i) examine how MI fits within international dietetic scope of practice, competency standards, and evidenced-based guidelines and (ii) provide recommendations for applying $\mathrm{MI}$ in dietetic practice and research.

\section{Description of the competency review}

How MI aligns with dietetic scope of practice and competency standards

United States (U.S.) ${ }^{22,23}$, Canadian $^{24}$, European ${ }^{25}$ and Australian ${ }^{26,27}$ dietetic scope of practice statements and competency standards were examined for MI themes using the definition and synonyms of core MI principles. Two of the four concepts, partnership and compassion, were consistent with all reviewed scope of practice statements and competency standards (table 1). All sources stated the importance of providing 'client-centered', 'collaborative' consultations that meet the needs and values of their clients. Acceptance was emphasized in the Canadian ${ }^{24}$ and European ${ }^{25}$ competency standards, with statements such as 'protects a client's right to autonomy’, 'respect for individual differences' and 'uses active listening techniques'. Whilst U.S. $^{22,23}$ and Australian ${ }^{26,27}$ standards did not explicitly state acceptance, autonomy was reflected under the MI construct of partnership such as 'confers with', 'negotiates' and ‘involves customers in decision making’. 
112 No statements documented the importance of evoking intrinsic motivation from clients. ${ }^{23-25,27}$

113 Intrinsic motivation is an autonomous form of motivation where the behavior is engaged

114 willingly due to the inherent satisfaction it holds for the individual. ${ }^{3}$ Rather than imposing

115 their views on the client, a dietitian encouraging intrinsic motivation would discuss behavior

116 change in relation to the client's values. ${ }^{3,15}$ Although the competency standards do not refer

117 to intrinsic motivation, this does not suggest that its evocation is outside the scope of the

118 dietetic profession, rather that it is not considered a minimum competency for graduate

119 dietitians internationally.

121 The dietetic standards, taken collectively, emphasized the importance of practitioners seeking new learning opportunities, engaging in professional development and self-review and integrating research findings into dietetic practice. ${ }^{22-27}$ Behavior change counseling has been recognized by dietitians and professional bodies as a major professional development need in recognition that additional skills may help clients achieve behavior change. ${ }^{12}$

128 The definition and description of MI clinical strategies were reviewed against the U.S. ${ }^{28,29}$, 129 Canadian $^{30}$, European ${ }^{31,32}$ and Australian ${ }^{12,33,34}$ weight management guidelines to determine their congruence with MI (table 2). The MI strategies of goal setting and developing a change plan were evident in all guidelines ${ }^{12,28-34}$, and would be considered congruent with MI if the goals and strategies are developed by the client with the dietitians support. Asking questions consistent with the MI strategy of importance and confidence rulers were recommended in 
134 the Australian guidelines. ${ }^{33}$ Whilst no guideline specifically outlined the use of these rulers, the activity aligns with each guideline's evidenced-based recommendation for assessing readiness to change. Decisional balances were directly mentioned by the Canadian guidelines only. $^{30}$ U.S. ${ }^{28,29}$, European ${ }^{31,32}$ and Australian ${ }^{12,33,34}$ guidelines stated the importance of eliciting willingness and reasons for change, but did not mention eliciting reasons for not making a change. Considering the advantages and disadvantages of change is important in resolving ambivalence in MI. ${ }^{3,15}$

Evidence-based recommendation for self-monitoring activities and barrier identification ${ }^{12,28-}$ ${ }^{34}$ are compatible with the action phase of MI. Best-practice clinical strategies may not be MIadherent if carried out before the client is ready. ${ }^{35}$ An example of this would occur if a dietitian promotes goal setting and developing a plan when the client is ambivalent or before they have expressed a commitment to change. If a dietitian chooses to motivate their clients using reward systems (such as prizes or gift certificates) ${ }^{28,36}$ or coaches clients using motivational techniques of persuasion, coercion or social pressure, then consultations would not be consistent with MI as these strategies promote external motivation through fear of failure, guilt or external pressure. ${ }^{3}$

Four categories of nutrition interventions were internationally recognized by the International Dietetic Nutritional Terminology Manual: i) food and/or nutrient delivery, ii) nutrition education, ii) nutrition counseling, and iv) co-ordination of nutrition care. ${ }^{36}$ Nutrition counseling may be conducted in an MI style if it aims to collaboratively establish priorities, goals and change plans that facilitate client responsibility for improving health outcomes. 
157 Whilst nutrition education can be carried out in the 'spirit' of MI, nutrition education would not be compliant if carried out in an instructing 'expert-recipient' manner, if the client is not yet ready to change, or if the client's permission to receive education has not been sought prior to the act of informing. ${ }^{1}$ Dietitians may find it challenging to accept the change in role from 'expert' to 'facilitator', especially if the dietitian and/or client are more familiar with traditional advice-giving models. Table 3 provides examples of the ways dietitians can incorporate MI-consistent communication into consultations.

\section{Implications for Dietetic Research}

Even though the efficacy and cost-effectiveness of MI in dietetic practice has not been established $^{19}$, evidence from MI interventions by other health professions warrants research examining the incorporation of MI into dietetic consultations. Researchers aiming to determine the effectiveness of MI interventions should evaluate it against an attention control. $^{21}$ In the absence of an attention control, it is impossible to determine if the intervention effect is due to MI or the amount of dietetic contact per se. ${ }^{21}$ One of the challenges in MI research is that intervention studies require a highly documented protocol, yet the constructs of MI support a flexible consultation directed by client needs. Adhering to the research protocol could initiate client resistance due to feeling pressured to move to the next stage before they are ready, while conversely, adhering to MI may introduce heterogeneity into the intervention content.

MI is not a set of clinical techniques or a protocol that is applied to clients, rather it is the 'spirit' of the entire conversation. ${ }^{35}$ It requires comprehensive training and evaluation ${ }^{35,37}$, 
which can be expensive and time-consuming. There is evidence that extensive MI training may be required to achieve proficiency ${ }^{35,37}$ based on evidence that immediate improvements following initial MI training are not usually maintained. ${ }^{38}$ Basic training may leave the health professional feeling overly confident in their MI skill level, or that they were already practicing according to MI principles. ${ }^{39}$ The timing and intensity of training required to achieve competency in all aspects of MI remains unknown. ${ }^{21}$ To ensure intervention fidelity it is crucial that researchers evaluate and report the competence of dietitians delivering the intervention using validated fidelity tools such as the Motivational Interviewing Treatment Integrity (MITI) ${ }^{40,41}$, or the Motivational Interviewing Screening Code (MISC). ${ }^{42}$

\section{Implications for Dietetic Practice}

The scope of practice statements, competency standards and evidenced-based guidelines evaluated predominately aligned with MI. While this does not necessarily mean dietitians are practicing according to these principles, it is encouraging that training in $\mathrm{MI}$ is compatible with international dietetic practice standards. However, considering that two to three days of training has been found to be insufficient in achieving competency ${ }^{11,16,17,39}$, 'pure' MI may not be achievable for dietitians not already skilled in advanced counseling or not willing to engage in extensive and continual training. ${ }^{21}$

Training in MI increases the skill set of a dietitian by providing effective, evidence-based communication skills and clinical strategies to guide behavior change discussion. ${ }^{3} \mathrm{MI}$ allows dietitians to distinguish between preparatory and commitment stages of change (figure 1) and to tailor discussion to either facilitate the client to strengthen their commitment or commence 
203 developing a change plan. There is no evidence to indicate that MI is harmful, even if not 204 carried out to a high proficiency level. It is highly recommended that the practitioner 205 demonstrates and documents competence in the area of MI and nutrition education and 206 counseling in general prior to utilizing the MI method. Introductory MI training may still 207 foster a more collaborative, client-centered consultation compared to advice-giving. ${ }^{6}$ 208 Dietitians interested in learning more about MI could:

- Familiarize themselves with MI principles, communication language and clinical strategies through books ${ }^{1,3,15}$, journal articles ${ }^{6,35,43,44}$ and information by the Motivational Interviewing Network of Trainers $(\mathrm{MINT})^{45}$, an international organization of MI trainers.

- Participate in MI workshops with qualified trainers advertised through the $\mathrm{MINT}^{45}$ or

- Practice MI with appropriate clients. After obtaining client and employer consent, record consultations and replay to identify areas of improvement, or seek feedback from colleagues for objective feedback.

- Be involved in researching the efficacy and cost-effectiveness of MI as an intervention in the dietetic profession.

- Repeat the above mentioned steps. As the authors of the counseling style state: learning MI is 'a process, not a curriculum”. 3(p192) It may take years to become proficient so it should be viewed as a long term investment in quality counseling. 


\section{Conclusion}

226 MI was not designed as a 'blanket' counseling strategy for all clients. ${ }^{35}$ While many clients 227 may prefer a non-prescriptive guiding approach (such as MI), some clients will prefer a more 228 direct, advice-giving consultation ${ }^{6,7}$, particularly when ambivalence to change is low and few 229 barriers are identified. Dietitians need to be alert, flexible and responsive to behavior change 230 evidence and client needs, to ensure that effective counseling methods are being 231 employed. ${ }^{8,22-27} \mathrm{MI}$ is one counseling style in the array of counseling models applicable to 232 dietitians that requires further research. 


\section{References}

235

1. Rollnick S, Miller, W., Butler, C. Motivational Interviewing in Health Care: Helping patients change behaviour. New York: Guilford Press; 2008.

2. Fjeldsoe B, Neuhaus M, Winkler E, Eakin E. Systematic review of maintenance of behavior change following physical activity and dietary interventions. Health Psychol. Jan 2011;30(1):99-109.

3. Miller WR, Rollnick, S. Motivational Interviewing: Preparing people for change. 2nd ed. New York: The Guilford Press; 2002.

4. Rollnick S, Kinnersley P, Stott N. Methods of helping patients with behaviour change. Br Med J. Jul 17 1993;307(6897):188-190.

5. Thomas B. Manual of Dietetic Practice, 2nd Edition. Cambridge: The University Press; 1994.

6. Rollnick S, Butler CC, McCambridge J, Kinnersley P, Elwyn G, Resnicow K. Consultations about changing behaviour. Br Med J. 2005;331(7522):961.

7. Hancock RE, Bonner G, Hollingdale R, Madden A. 'If you listen to me properly, I feel good': a qualitative examination of patient experiences of dietetic consultations. $J$ Hum Nutr Diet. 2012;25(3):275-284.

8. Spahn JM, Reeves RS, Keim KS, et al. State of the evidence regarding behavior change theories and strategies in nutrition counseling to facilitate health and food behavior change. J Am Diet Assoc. Jun 2010;110(6):879-891.

9. MacLellan D, Berenbaum S. Canadian dietitians' understanding of the client-centered approach to nutrition counseling. J Am Diet Assoc. 2007;107(8):1414-1417.

10. Cant R, Aroni R. Exploring dietitians' verbal and nonverbal communication skills for effective dietitian-patient communication. J Hum Nutr Diet. 2008;21(5):502-511. 
11. Brug J, Spikmans F, Aartsen C, Breedveld B, Bes R, Fereira I. Training dietitians in basic motivational interviewing skills results in changes in their counseling style and in lower saturated fat intakes in their patients. J Nutr Educ Behav. 2007;39:8-12.

12. Dietitians Association of Australia. DAA Best Practice Guidelines for the Treatment of Overweight and Obesity in Adults. Canberra: Dietitians Association of Australia; 2012.

13. Armstrong $\mathrm{M}$, Mottershead $\mathrm{T}$, Ronksley $\mathrm{P}$, Sigal R, Campbell $\mathrm{T}$, Hemmelgarn B. Motivational interviewing to improve weight loss in overweight and/or obese patients: a systematic review and meta-analysis of randomized controlled trials. Obes Rev. 2011;12(9):709-723.

14. Rubak S, Sandbaek A, Lauritzen T, Christensen B. Motivational interviewing: a systematic review and meta-analysis. Br J Gen Pract. 2005;55:305-312.

15. Miller WR, Rollnick S. Motivational Interviewing: Helping people change. 3rd ed. New York: Guilford Press; 2012.

16. Carels RA, Darby L, Cacciapaglia HM, et al. Using motivational interviewing as a supplement to obesity treatment: A stepped-care approach. Health Psychology. May 2007;26 (3):369-374.

17. Bennett JA, Young HM, Nail LM, Winters-Stone K, Hanson G. A telephone-only motivational intervention to increase physical activity in rural adults: A randomized controlled trial. Nurs Res. January/February 2008;57 (1):24-32.

18. Heinrich E, Candel M, Schaper NC, de Vries NK. Effect evaluation of a Motivational Interviewing based counselling strategy in diabetes care. Diabetes Res Clin Pract. Dec 2010;90(3):270-278. 
19. Resnicow K, Davis R, Rollnick S. Motivational Interviewing for Pediatric Obesity: Conceptual Issues and Evidence Review. J Am Diet Assoc. 2006;106(12):2024-2033.

20. Bowen D, Ehret C, Pedersen $\mathrm{M}$, et al. Results of an adjunct dietary intervention program in the Women's Health Initiative. J Am Diet Assoc. 2002;102:1631-1637.

21. Hollis JL, Williams LT, Collins CE, Morgan PJ. Effectiveness of interventions using Motivational Interviewing for dietary and physical activity modification in adults: A Systematic Review. The Joanna Briggs Institute Database of Systematic Reviews and Implementation Reports. 2013;11(5):1-27.

22. The Academy Quality Management Committee and Scope of Practice Subcommittee of the Quality Management Committee. Academy of Nutrition and Dietetics: Scope of practice for the registered dietitian. J Acad Nutr Diet. 2013;113 (suppl 2):S17-S28.

23. The Academy Quality Management Committee and Scope of Practice Subcommittee of the Quality Management Committee. Academy of Nutrition and Dietetics: Revised 2012 standards of practice in nutrition care and standards of professional performance for registered dietitians. J Acad Nutr Diet. 2013;113 (suppl 2):S29-S45.

24. Dietitians of Canada and College of Dietitians of Ontario. Professional standards for $\begin{array}{llll}\text { dietitian } & \text { in } & \text { Canada. }\end{array}$ http://www.cdo.on.ca/en/pdf/publications/ProfessionalStandardsforDietitians.pdf. Accessed 16 August, 2013.

25. European Federation of the Association of Dietitians. European Dietetic Competences and their Performance Indicators2009.

26. Dietitians Association of Australia. Scope of dietetic practice framework. 2006; http://dmsweb.daa.asn.au/files/DINER/DINER_Scope_of_Practice.pdf. Accessed 16 August, 2013. 
27. Dietitians Association of Australia. National Competency Standards for entry level dietitians in Australia. 2008; http://daa.asn.au/universities-recognition/nationalcompetency-standards/. Accessed 16 August, 2013.

28. National Heart Blood and Lung Institute. Clinical Guidelines for the Identification, Evaluation and Treatment of Overweight and Obesity in Adults. Vol NIH Publication No. 98-4083: National Institutes of Health; 1998.

29. Seagle HM, Strain GW, Makris A, Reeves RS. Position of the American Dietetic Association: weight management. J Am Diet Assoc. Feb 2009;109(2):330-346.

30. Lau DC, Douketis JD, Morrison KM, Hramiak IM, Sharma AM, Ur E. 2006 Canadian clinical practice guidelines on the management and prevention of obesity in adults and children. Can Med Assoc J. 2007;176(8):1-117.

31. Tsigos C, Hainer V, Basdevant A, et al. Management of obesity in adults: European clinical practice guidelines. Obesity Facts. 2008;1(2):106-116.

32. National Institute for Health and Clinical Excellence. Obesity: Guidance on the prevention, identification, assessment and management of overweight and obesity in adults and children. London: National Institute for Health and Clinical Excellence; 2006.

33. National Health and Medical Research Council. Clinical Practice Guidelines for the Management of Overweight and Obesity in Adults, Adolescents and Children in Australia. Canberra: National Health and Medical Research Council; 2013.

34. Dietitians Association of Australia. Best Practice Guidelines for the Treatment of Overweight and Obesity in Adults. Canberra: Dietitians Association of Australia; 2005. 
35. Miller WR, Rollnick S. Ten things that motivational interviewing is not. Behavioural and cognitive Psychotherapy. Mar 2009;37(2):129-140.

36. Academy of Nutrition and Dietetics. International Dietetics \& Nutrition Terminology (IDNT) Reference Manual. ,Fourth Edition ed: Academy of Nutrition and Dietetics; 2013.

37. Miller WR, Yahne CE, Moyers TB, Martinez J, Pirritano M. A randomized trial of methods to help clinicians learn motivational interviewing. J Consult Clin Psychol. Dec 2004;72(6):1050-1062.

38. Martino S, Ball SA, Nich C, Frankforter TL, Carroll KM. Community program therapist adherence and competence in motivational enhancement therapy. Drug alcohol depend. 2008;96(1-2):37-48.

39. Miller WR, Mount KA. A small study of training in motivational interviewing: Does one workshop change clinician and client behavior? Behavioural and cognitive Psychotherapy. 2001;29(04):457-471.

40. Moyers T, Martin, T., Manuel, J., Miller, W., Ernest, D. Revised Global Scales: Motivation Interviewing Treatment Integrity 3.0 (MITI 3.0): University of New Mexico, Centre on Alcoholism, Substance Abuse and Addictions; 2007.

41. Moyers TB, Martin T, Manuel JK, Hendrickson SM, Miller WR. Assessing competence in the use of motivational interviewing. J Subst Abuse Treat. Jan 2005;28(1):19-26.

42. Miller WR, Moyers TB, Ernst D, Amrhein P. Manual for the Motivational Interviewing Skill Code (MISC) Version 2.0: University of New Mexico, Center on Alcoholism, Substance Abuse and Addictions; 2003. 
43. Rollnick S, Butler CC, Kinnersley P, Gregory J, Mash B. Motivational interviewing. Br Med J. 2010-04-27 00:00:00 2010;340.

44. Resnicow K, McMaster F. Motivational Interviewing: moving from why to how with autonomy support. International Journal of Behavioral Nutrition and Physical Activity. 2012/03/02 2012;9(1):1-9.

45. Motivational Interviewing Network of Trainers. Motivational Interviewing. 2013; http://www.motivationalinterviewing.org/home. Accessed 5th September, 2013. 